\title{
ELECTRON-ELECTRON SCATTERING INFLUENCE ON HOT ELECTRON TRANSPORT IN SEMICONDUCTORS
}

\author{
S. Dedulewicz AND Ž. KanCleris \\ Semiconductor Physics Institute, Lithuanian Academy of Sciences \\ A. Goštauto 11, 232600 Vilnius, Lithuania
}

\begin{abstract}
Electron-electron scattering has been shown to manifest itself when scattering by optical phonons is of importance. The strongest influence has been observed in the slightly heated electron system at the lattice temperature $T \approx T_{0} / 5$ ( $T_{0}$ being the characteristic temperature of optical phonon).
\end{abstract}

PACS numbers: $72.20 . \mathrm{Ht}$

It is known [1] that electron-electron scattering can increase hot electron excess energy dissipation and in this way influence its distribution function and parameters measured experimentally. This effect can be realized, when both ee-scattering and optical scattering is significant in electron system. Although ee-scattering mechanism has been widely investigated [2-5], the conditions at which the greatest influence of inter-particle collision can be observed has not been yet determined.

In the present paper ee-scattering influence on electron mean energy change in slightly heating electric field (warm electrons) was investigated. The conditions at which ee-scattering influences significantly warm electron parameters were established. Electron system energy losses were considered as well. The investigation was carried out up to electron concentration when ee-scattering and energy relaxation via electron-phonon scattering is of the same efficiency.

To calculate electron system mean energy and power losses in heating electric field the symmetrical part of the distribution function is to be known. In warm electron region it can be written down in the following way:

$$
f(\varepsilon, t)=f_{0}(\varepsilon)+\left(\frac{E}{E^{*}}\right)^{2} \delta f(\varepsilon, t)
$$

where $f_{0}$ and $\delta f$ are the equilibrium distribution function and $E^{2}$ correction to it, respectively, and $E^{*}$ is a constant measured in the units of electric field. Making use 
of dimensionless energy $x=\varepsilon / k T$ and the function $F(x, t)=\sqrt{x} \delta f(x, t)$, which describes particle density on the energy axis, kinetic equation for the function $F$ can be represented as [6]:

$$
\frac{\partial}{\partial t} F(x, t)=\sqrt{x} S_{0}\left(\frac{F}{\sqrt{x}}\right)-\frac{\partial}{\partial x}\left[x^{3 / 2} \tau(x) \exp (-x)\right]
$$

where $S_{0}$ is the symmetrical part of the collision operator and $\tau(x)$ is a properly normalized momentum relaxation time. Having solved equation (2) and obtained function $F(x)$, warm electron mean energy is to be expressed in the following way:

$$
\langle\varepsilon\rangle=\frac{3}{2} k T\left[1+\left(\frac{E}{E^{*}}\right)^{2} \frac{4}{3 \sqrt{\pi}} \int_{0}^{\infty} \mathrm{d} x x F(x)\right] .
$$

We have solved Eq. (2) and calculated the average electron energy in two limiting cases. First, ee-scattering was neglected and second, that scattering mechanism was supposed to dominate in Eq. (2). In the first case we used the two-particle Monte Carlo method proposed in [6], while in the second one the electron temperature approximation was employed. The ratio

$$
\eta=\frac{\langle\varepsilon\rangle-\frac{3}{2} k T}{\langle\varepsilon\rangle_{\mathrm{m}}-\frac{3}{2} k T}
$$

dependence on inverse temperature $u=T_{0} / T$ was calculated. Here $\varepsilon$ and $\langle\varepsilon\rangle_{\mathrm{m}}$ represent electron average energy in electric field calculated without allowance for ee-scattering and in the case when this scattering mechanism dominates. Thus, $\eta$ value shows how much ee-scattering can influence the mean electron energy in a heating electric field.

Results of calculations for $p$-Ge, $n$-InSb and $n$-GaAs are presented in Fig. 1 . It is seen that the dependence of the parameter $\eta$ on $u$ is non-monotonous and reaches its maximum at $u \approx 5.4$ in all materials under consideration. A very strong influence of ee-scattering on mean electron energy is observed in polar semiconductors. It should be pointed out that $\eta$ maximum position is independent of the type of electron interaction with optical phonons (polar or deformation potential). Moreover, linear dependence of $\eta(5.4)$ on $W=S_{0}^{(o)} / S_{0}^{(a)}$, where $S_{0}^{(o)}$ and $S_{0}^{(a)}$ are the constants defining optical and acoustic scattering intensity in Eq. (2), is observed. The additional investigation has also revealed that the increase of electric field leads to the decrease of $\eta$.

The explanation of ee-scattering influence on electron system, when scattering by the optical phonons is significant, is usually based on the assumption that inter-particle collisions condition increase of the optical phonon scattering intensity (so-called complex scattering mechanism [1]). Thus, the change of electron system power losses may be observed. To calculate them energy balance equation was used.

Calculation results for $n$-GaAs are shown in Fig. 2. Here the total power losses and their components dependencies on the temperature are presented. It is seen that ee-scattering increases power losses conditioned by optical scattering mechanism and decreases losses conditioned by acoustic scattering. Optical 


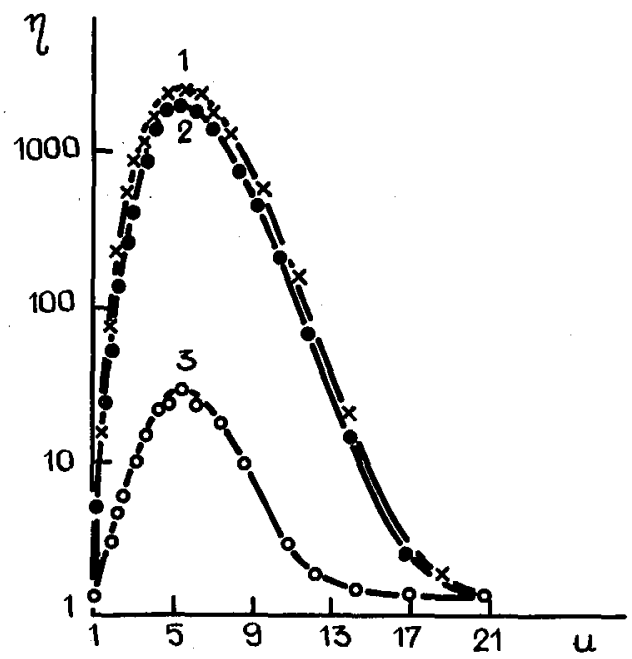

Fig. 1. $\quad \eta$ dependence on inverse temperature $u=T_{0} / T$, where $T_{0}$ is characteristic temperature of the optical phonon. Curves 1,2 and 3 correspond to $n$-InSb, $n$-GaAs and $p$-Ge, respectively.

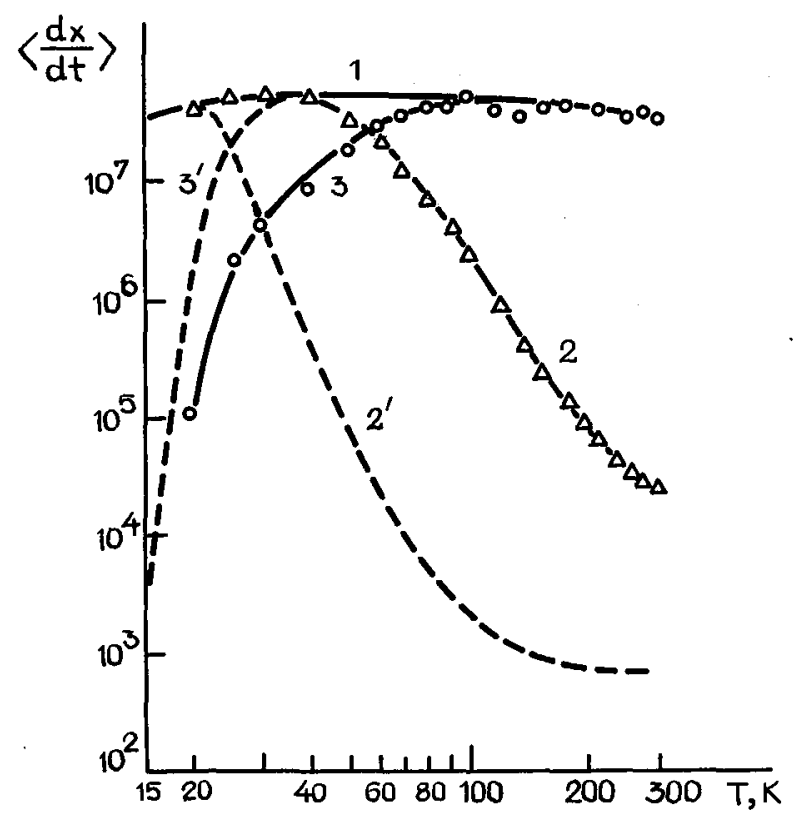

Fig. 2. Total power losses (curve 1) and their components (conditioned by scattering on acoustic (curves $2,2^{\prime}$ ), and optical phonons (curves $\left.3,3^{\prime}\right)$ ) dependencies on temperature in $n$-GaAs. Curves 2,3 and $2^{\prime}, 3^{\prime}$ are calculated without allowance for ee-scattering and in the case when this scattering mechanism dominates, respectively. 
scattering intensity increase can be interpreted as the evidence of complex scattering mechanism [1] in the warm electron region. It should be pointed out that ee-scattering does not change total power losses (curve 1), because it is proportional to Joule heating $\sim \mu_{0} E^{2}$ and mobility $\mu_{0}$ is not influenced by ee-scattering in the concentration range under consideration. In spite of this, partial power losses redistribution conditioned by ee-scattering will be sufficient to change the warm electron distribution function and other warm electron parameters.

In conclusion, we wish to emphasize that maximum influence of ee-scattering on electron system which interacts with optical phonons is observed in warm electron region at the temperature $T \approx T_{0} / 5$.

\section{References}

[1] I.B. Levinson, G.E. Mažuolyté, Zh. Eksp. Teor. Fiz. 50, 1048 (1966) [JETP 23, $697(1966)]$.

[2] A. Matulionis, Yu. Pozhela, A. Reklaitis, Solid State Commun. 16, 1133 (1975).

[3] P. Lugly, D.K. Ferry, Physica B 134, 364 (1985).

[4] S. Dedulevich, Ž. Kancleris, A. Matulis, Fiz. Tekh. Poluprov. 22, 881 (1988) [Sov. Phys. Semicond. 22, 556 (1988)].

[5] S. Dedulevich, Ž. Kancleris, A. Matulis, Zh. Eksp. Teor. Fiz. 95, 1701 (1989) [JETP 68, 982 (1989)].

[6] Ž. Kancleris, A. Matulis, J. Phys. C: Sol. St. Phys. 20, 1273 (1987). 